\title{
No Pathologic Evidence of Distant Metastasis
}

National Cancer Institute

\section{Source}

National Cancer Institute. No Pathologic Evidence of Distant Metastasis. NCI Thesaurus.

Code C158827.

A result that there is no pathologic evidence of distant metastasis. 